\title{
Heiko Klinge Mögliche Konsequenzen für die kirchliche Presse
}

Wie kann man mit den Erkenntnissen aus den Sinus-Milieus im Alltag von Redaktionen und Verlagen kirchlicher Presse umgehen? Darüber soll ich schreiben, so die Herausgeber von „Communicatio Socialis“. Warum gerade ich? Das war meine Frage nach der Lektüre des Milieu-Handbuches der MDG und des Katholischen Sozialethischen Instituts KSA. Denn die Lektüre des umfangreichen Bandes war für den Nichtsoziologen und Nichtpsychologen eine trockene, zum Teil mühevolle Sache.

Und was sage ich nun meinen Kollegen in den Chefredaktionen oder Verlagsleitungen als früherer Verleger einer Kirchenzeitung, was sagt der frühere Geschäftsführer einer Unternehmensberatung den Kunden der MDG? Was den Medienverantwortlichen im Verband der Diözesen Deutschlands und in den Diözesen, die diesen Aufsatz eventuell auch zu Gesicht bekommen?

Schlaue Rezepte, wie Themen, Texte, in welchen journalistischen Formen für welche Milieus zu veröffentlichen sind, wie sie und wo und wann zu platzieren sind, Rezepturen, die man nur anzuwenden braucht, um erfolgreich Auflagen und Umsätze zu erhöhen, wird man in Folgendem nicht finden.

Was würde ich nach der Lektüre der Studie machen, wäre ich heute noch Verleger oder Chefredakteur, was würde ich als Berater heute meinen Kunden sagen? Das allerdings will ich gerne darzustellen versuchen.

\section{Soziodemographische Daten}

Zunächst würde ich meine eigenen Daten mit denen der quantitativen Studie „MDG/IfD-Trendmonitor Religiöse Kommunikation 2003“ und der qualitativen Sinus-Milieustudie gegenüberstellend abgleichen. Falls Daten wie Alter, Bildung, Beruf, Einkommen, Wohnquartier usw. in meinem Unternehmen nicht vorhanden sind, wäre das eine nicht einfache, nicht preiswerte, aber eine notwendige Arbeit, sie schnellstmöglich $\mathrm{zu}$ beschaffen. Wenn meine Kollegen in anderen Verlagen ähnliche Probleme hätten, würde ich versuchen, gemeinsam zu handeln oder die MDG bitten, mir zu helfen.

Zum Beispiel würde ich gerne wissen wollen, in welchen Orten und Stadtteilen, ja Straßen meines Verbreitungsgebietes welche der Milieus wohnen. Kann Sinus mir hier helfen? Ist das Seelsorgereferat meiner Diözese daran eventuell auch interessiert? 


\section{Lebensweltanalyse}

Ich würde Fachleute bitten (Journalisten, Theologen, Soziologen, Betriebswirte) eine Synopse zu erstellen und somit Erkenntnisse aus meiner letzten eigenen Leseranalyse, aus dem „Trendmonitor Religiöse Kommunikation 2003“, aus Copytests und/oder eventuell ReaderScanErkenntnissen den Äußerungen aus den Milieus gegenüber zu stellen und abzugleichen.

Wäre mein Verlag, wäre meine Redaktion personell - aufgrund der Größe - dazu nicht in der Lage, würde ich auch hier um Hilfe der Berater bitten und/oder mit Nachbarn zusammenarbeiten.

\section{Abgleich des Medienverhaltens}

Die Affinität der zehn Milieus zu Printprodukten und Rundfunkangeboten würde ich untereinander vergleichen lassen und mit dem Medienverhalten der Katholiken und meiner Leser aus dem vorhandenen Material (Leseranalysen, Trendmonitor, Rundfunkgutachten) abgleichen.

Da ich die Themen und die "Schreibe“ der „Konkurrenz" kenne, würde ich überprüfen, ob „meine Schreibe“, ob meine Themen damit korrespondieren oder nicht.

\section{Abgleichung der Sinusbeschreibung der sozialen Milieus $A, B$ und $C$}

Ich würde - vorrangig - die Äußerungen zu Lebenssinn, Weltanschauung, Religion und Kirche, Bibel, Image der Kirche und Wünsche an die Kirche aus den zehn Sinus-Milieus in Rastern systematisch und möglichst genau abgleichen, um eventuelle Übereinstimmungen oder ähnliche Tendenzen bzw. Differenzen und entgegengesetzte Tendenzen feststellen zu können.

Nachrangig würde ich dann die zehn Sinus-Milieus nach Lebensstil, Alltagsästhetik, sozialer Identität, Freizeit, Sprache und Sehnsüchten gegenüberstellen. Eventuell kann ich neben redaktionellem Nutzen einen wirtschaftlichen Nutzen daraus für die Anzeigen- und Beilagenakquisition ziehen.

$\mathrm{Zu}$ den Punkten 3. und 4. wäre eine Beauftragung der MDG sicher hilfreich und für mich selbst - wäre ich Verleger oder Chefredakteur sehr zeitsparend.

$\mathrm{Zu}$ Punkt 3. würde mich besonders das Online-Verhalten bzw. die Online-Affinität interessieren, und wenn ich das Online-Verhalten meiner Leser nicht kenne, würde ich sie schnell befragen, denn dieses Thema ist für die nahe Zukunft meiner Kirchenzeitung ohnehin von großer Wichtigkeit. 
5. Erkenntnisse aus den Punkten 1. bis 4.

Nun müssten meine Kollegen und ich mit der MDG eine Arbeitsgruppe bilden. Dort sollten die Konsequenzen, die sich grundsătzlich für Printmedien ergeben, gemeinsam erarbeitet und konkrete Maßnahmen vorgeschlagen werden.

Alle Erkenntnisse, die sich für meine Zeitung oder Zeitschrift ergeben, würde ich mit meinen Mitarbeitern unter Moderation eines Beraters in Maßnahmen für meine eigene Zeitung umwandeln. Hier würde ich auch je einen Experten aus der Pastoralabteilung und aus der Abteilung für Öffentlichkeitsarbeit meiner Diözese hinzunehmen.

\section{Eine Spezialität für die Bistumspresse}

Da die Bistumszeitungen aufgrund der jeweiligen geographischen Alleinstellung keine Konkurrenten sind, könnten sie gemeinsam eine ganz andere Überlegung anstellen, die sich vordergründig nicht aus der Milieu-Studie ergibt, bei näherem Hinsehen jedoch wohl.

Aufgrund des Digitaldruckes gibt es preiswerte Möglichkeiten, auch schon kleinste Auflagen herzustellen und diese mit Teilen der Gesamtauflage zusammenzubringen. Als Verleger oder als Chefredakteur würde ich mir überlegen, ob man dadurch nicht zu der ohnehin wichtigen Regionalberichterstattung im Bistumsteil auch noch eine Lokalberichterstattung für Gemeinden organisieren könnte und - vielleicht ein wenig mutig - sogar für bestimmte Milieus. Dass diese Frage erst beantwortet werden kann, wenn alle Voraussetzungen von redaktioneller Leistungsfähigkeit, Herstellung und Vertrieb geklärt sein müssen, ist klar.

Wenn man aber ein von der Fläche her überschaubares Bistum während seiner Strukturreform, aus vielen Gemeinden wenige machen zu müssen, als Modellbistum nutzen dürfte, um diese Frage konkret prüfen und eventuell sogar einige Monate ausprobieren könnte, damit auch andere Diözesen Nutzen daraus ziehen, so wäre es den Schweiß der Edlen wert. Eventuell stellt sich jedoch heraus, dass es ein Traum war. Aber das Risiko scheint mir gering zu sein.

Darüber hinaus könnte man Abonnenten aus bestimmten Milieus zusätzlich Online-Bistumszeitungen anbieten. Dieser Online-Zusatznutzen müsste die Interessen der jeweiligen Abonnentengruppen berücksichtigen. Auch ein solches Modell könnte konzipiert, experimentell umgesetzt und wissenschaftlich begleitet werden in einem Bistum. Als 0nline-Modell.

Für ein Modell mit Digitaldruck-Beilagen und ein Online-Modell benötigt man die fachliche und finanzielle Hilfe der MDG und ein interessiertes Bistum, dem man bei den schwierigen Aufgaben der Neuordnung der Gemeinden eine Hilfe bieten könnte. So wäre der Bistumszeitung 
geholfen, der Pastoral in Gemeinden und Diözese und - je nach Ergebnis des Modellversuches - auch anderen Presseorganen, die von dem Modell profitieren könnten.

\section{Zeitfaktor}

Damit die Maßnahmen möglichst zeitnah - allerdings ohne Hektik begonnen werden können, sollte ein Ablaufplan mit Parallelschritten erstellt werden, mit klar definierter Priorisierung.

\section{Nutznießer der Ergebnisse}

Nutznießer sind alle Presse-Verlage mit Objekten in unterschiedlichster Erscheinungsweise (z. B. Fachpresse, Wochenpresse und sonstige Periodika) in unterschiedlichster Funktion und in welcher Trägerschaft auch immer, ob in kirchlichem Eigentum oder im Besitz privater Verleger.

Wenn ich - wäre ich noch Verleger - trotz aller damit verbundenen Schwierigkeiten die sich eventuell herausstellende Chance der MilieuStudie nicht nutzte, dann würde ich meinem Job nicht gerecht werden.

Ganz sicher würde ich mich von Bedenkenträgern - uninformiert, wie sie oft sind - nicht irritieren lassen. Die Sinus-Milieustudie - übrigens von der Publizistischen Kommission der Deutschen Bischofskonferenz gewollt, von der Koordinierungskommission des Verbandes der Diözesen Deutschlands beschlossen und von der MDG in Auftrag gegeben - ist natürlich nicht das Evangelium. Aber sie ist ein sehr wichtiges Mittel zur Erweiterung der eigenen Erkenntnis und eine hervorragende Anregung, das als richtig Erkannte auch umzusetzen.

Nach Lektüre der Studie und nach der näheren Beschäftigung mit der Materie kann ich nur sagen, schade, dass ich kein Verleger mehr bin. Gut aber, dass ich mich - dank der Anfrage von "Communicatio Socialis" damit beschäftigen durfte. Ich bin sehr nachdenklich geworden und habe wieder einmal dazugelernt. Ich hoffe, es geht vielen meiner ehemaligen Kollegen, die noch Entscheidungen treffen können, ebenso. Dann werden sich in kirchlichen Printmedien gewiss - hier und dort - Veränderungen erkennen lassen, zum Nutzen der kirchlichen Presse, der Pastoral und damit der Menschen. 